\title{
NORMAL MODE ANALYSIS OF VIBRATIONS OF JET-COOLED ACRIDINE DIMER
}

\author{
I. Deperasińska and J. Prochorow \\ Institute of Physics, Polish Academy of Sciences \\ Al. Lotników 32/46, 02-668 Warsaw, Poland
}

(Received November 19, 1999)

\begin{abstract}
The analysis of normal modes of vibrations was performed for acridine dimer, which has been recently observed and identified by laser-induced emission spectroscopy under conditions of jet-cooling in a supersonic helium expansion. The frequencies and the forms of normal vibrations, computed with the use of the semi-empirical PM3 method are classifying all vibrations of acridine dimer into two distinct categories of inter- and intramolecular vibrations. There are six low-frequency intermolecular normal vibrations which are characterizing relative movements of two acridine moieties in the dimer and at least two of them have vibrational frequencies which are close to the frequencies of vibronic bands observed in the fluorescence excitation spectrum of the dimer. The intramolecular normal vibrations of the dimer very strictly correspond to the normal vibrations of acridine molecule (with a minor modification of the vibrational frequencies due to the splitting in the dimer). Furthermore, the intramolecular vibrations of acridine dimer, as well as the vibrations of acridine molecule are in good agreement with available experimental data (either IR and Raman spectra of acridine or fluorescence excitation spectrum of acridine dimer in supersonic jet). The results of the present analysis are very clearly supporting the previous conclusions concerning the ground-state equilibrium structure of acridine dimer formed under jet-cooling conditions.
\end{abstract}

PACS numbers: 33.20 .Tp

\section{Introduction}

The reports on observation of the formation of weakly bound molecular self-complexes (or Van der Waals type homo-dimers) of aromatic hydrocarbon systems and their various derivatives under conditions of supersonic jet cooling were increasing in number during the last decade (for a review see [1]). As a rule, a supersonic molecular beam contains a monomer/dimer mixture and the observed fluorescence and fluorescence excitation spectra of the expansion are composed of 
the overlapping monomer and dimer spectra (of different dependence of their intensities on the partial pressure of the molecules under study in the expansion). Usually, an excitation spectrum of laser-induced-fluorescence (LIF) of the dimer has the vibronic origin shifted towards lower energies as compared to the spectrum of bare (monomer) molecule and contains some additional low-frequency vibronic transitions characteristic of the intramolecular vibrations of the dimer which are not present in the parent molecule. If the dimer geometry can be predicted or determined then a normal mode analysis may be used for the assignments of these intermolecular modes.

Recently, in the course of laser-induced emission studies of jet-cooled acridine (ACD) molecules we have found that in a helium supersonic jet seeded with ACD molecules the only observed emission is fluorescence of acridine dimers, $(\mathrm{ACD})_{2}$, which are being formed in the expansion [2]. Acridine molecules by themselves are not fluorescing under these conditions which is not completely unexpected in view of the well-known sensitivity of the photophysics of acridine to environmental conditions [3].

The ground-state geometry of $(\mathrm{ACD})_{2}$ determined initially by the AM1 calculations [2] and verified by the PM3 method [4] is a head-to-tail structure with practically coplanar molecular planes and almost collinear arrangement of the short molecular axes of both ACD moieties (cf. Fig. 1). Such a structure of (ACD) dimer, in which the nitrogen atom of one ACD molecule is located directly above $\mathrm{C}-\mathrm{H}$ bond of the central ring of the second ACD molecule, provides a large enhancement of the oscillator strength for the transition from the ground to the first excited singlet state of the dimer [2,4]. It also indicates that the formation of $\mathrm{N}$..H-C hydrogen-bond between the two ACD moieties can play a role in stabilization of $(\mathrm{ACD})_{2}$, as observed in some other cases of aza-aromatic compounds (e.g. [5]), and clearly demonstrated by recent observations of the formation of strongly fluorescent hydrogen-bonded complexes of acridine with methanol in the supersonic beam [4].

A preliminary analysis of the observed vibronic structure of LIF excitation spectra of $(\mathrm{ACD})_{2}$ [2] was based on a comparison with the data available from the observations of the ground-state IR and Raman spectra in liquid and in solid phase $[6,7]$ and from the analysis of the vibronic structure of electronic absorption and phosphorescence spectra in low-temperature crystalline and amorphous matrices $[8,9]$. This comparative analysis has shown that the observed structure of LIF excitation spectrum can be separated into two sets of vibrational modes - the low-frequency vibrational modes of clear intermolecular origin inherent to $(A C D)_{2}$ and the vibrational modes which presumably are of intramolecular origin.

This paper is aimed at a quantitative analysis of vibrations (frequencies as well as normal mode description) in the ground state of (ACD) $)_{2}$ and ACD monomer. Such an analysis should provide the necessary criterions for the distinction between the intermolecular vibrational modes connected with the formation of $(\mathrm{ACD})_{2}$ in the supersonic expansion and intramolecular vibrations of $A C D$ monomers bound in the dimer. Furthermore, the results of the normal modes analysis can be utilized, at least qualitatively, for the interpretation and assignment of the vibronic structure of the observed fluorescence excitation spectra. 


\section{Vibrational analysis of acridine molecule and acridine dimer}

The normal modes analysis of the vibrations of ACD molecule and of its dimer was performed utilizing a semiempirical PM3 method [9] which, despite some obvious deficiencies, is generally considered as suitable for handling the vibrational problems in weakly bound molecular and hydrogen-bound complexes and which we have utilized earlier for optimization of the ground-state geometry of acridine dimer and calculations of its molecular orbitals, electronic states, and relevant transition dipole moments [4].

The results of the normal modes analysis for $(A C D)_{2}$ dimer, obtained by the PM3 [10] with HyperChem 5.1. computational package, are collected in Table. Calculations were performed for the PM3-optimized ground-state structure of $(A C D)_{2}$ [4] which is illustrated in Fig. 1. Although in the arbitrary chosen perspective projection in Fig. 1 this structure looks as coplanar and collinear (along the short molecular axes of both ACD moieties) there are some slight deviations from coplanarity and collinearity. In fact, molecular planes of $A_{1}$ and $A_{2}$ moi-
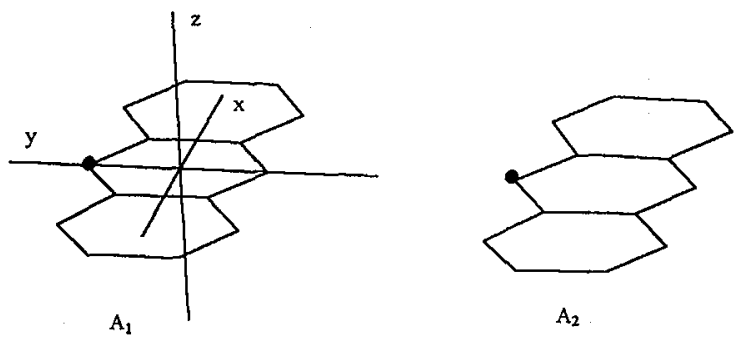

Fig. 1. PM3 optimized structure of the ground state of acridine dimer in an arbitrary chosen perspective projection. The cartesian coordinate system is fixed centrally at $A_{i}$ acridine moiety ( $x y$-plane of coordinate system coincides with the molecular plane of $\left.A_{1}\right)$. Black dots in the central rings of acridine moieties are representing the nitrogen atoms.

eties are rotated by $5^{\circ}$ about $x$-axis and by $\sim 1^{\circ}$ about $y$-axis (of the Cartesian coordinate system fixed at $A_{1}$ in Fig. 1). Hence, the dimer of acridine has no symmetry elements other than identity ( $C_{1}$ point group of symmetry) and the analysis does not yield the symmetry assignments for calculated normal vibrational modes. Table contains also the results of the PM3 normal mode analysis for ACD molecule, together with the symmetry of vibrational modes (under symmetry of $C_{2 v}$ point group of ACD).

The comparison of vibrational frequencies computed for ACD molecule and $(\mathrm{ACD})_{2}$ dimer, collected in Table, shows that the spectrum of vibrations of $(\mathrm{ACD})_{2}$ contains many more frequencies than that of $\mathrm{ACD}$. Furthermore, it is clearly seen that all vibrations in $(A C D)_{2}$ can be categorized in two sets (contrary to those found for ACD). First of all there are six low-frequency vibrations which are not present in ACD molecule and secondly all vibrations found for ACD can also be traced in $(\mathrm{ACD})_{2}$ but they are of a "doublet" character. 
TABLE

PM3 computed frequencies of the normal vibrations of acridine and acridine dimer.

\begin{tabular}{|c|c|c|}
\hline \multicolumn{2}{|c|}{$\begin{array}{c}\text { Computed frequency of normal } \\
\text { vibrations }\left[\mathrm{cm}^{-1}\right]\end{array}$} & \multirow[t]{2}{*}{$\begin{array}{l}\text { Vibrational frequency }{ }^{a} \text { observed for acridine molecule } \\
\text { and acridine dimer }\end{array}$} \\
\hline Acridine & Acridine dimer & \\
\hline & $\begin{array}{r}5 \\
12 \\
21 \\
39 \\
105 \\
113\end{array}$ & $\begin{array}{l}26,34,46,74,144,152 \\
\text { vibronic transitions observed in acridine dimer fluorescence } \\
\text { excitation spectrum (frequency shift from the vibronic ori- } \\
\text { gin cf. Fig. 5A) [2] }\end{array}$ \\
\hline $87\left(b_{1}\right)$ & $\begin{array}{l}87 \\
87\end{array}$ & $108[6]$ \\
\hline $101\left(a_{2}\right)$ & $\begin{array}{l}100 \\
102\end{array}$ & . \\
\hline $212\left(a_{2}\right)$ & $\begin{array}{l}213 \\
214\end{array}$ & $202[2], 237[6]$ \\
\hline $236\left(b_{1}\right)$ & $\begin{array}{l}236 \\
238\end{array}$ & \\
\hline $248\left(a_{1}\right)$ & $\begin{array}{l}248 \\
250\end{array}$ & $243[2], 240,237[6]$ \\
\hline $330\left(b_{1}\right)$ & $\begin{array}{l}331 \\
333\end{array}$ & $282 ?[2], 275[6], 285[7]$ \\
\hline $417\left(a_{1}\right)$ & $\begin{array}{l}417 \\
420\end{array}$ & $392[2], 401[6], 395,402[7]$ \\
\hline $426\left(b_{2}\right)$ & $\begin{array}{l}427 \\
433\end{array}$ & $417,414[6], 416[7]$ \\
\hline $451\left(a_{2}\right)$ & $\begin{array}{l}451 \\
452\end{array}$ & \\
\hline $462\left(a_{2}\right)$ & $\begin{array}{l}463 \\
465\end{array}$ & \\
\hline $464\left(b_{1}\right)$ & $\begin{array}{l}465 \\
468\end{array}$ & $469,476[6], 480[7]$ \\
\hline $516\left(b_{1}\right)$ & $\begin{array}{l}516 \\
517\end{array}$ & \\
\hline $539\left(b_{2}\right)$ & $\begin{array}{l}539 \\
540\end{array}$ & $523,521[6], 520[7]$ \\
\hline $604\left(b_{2}\right)$ & $\begin{array}{l}604 \\
625\end{array}$ & $600[6], 600[7]$ \\
\hline $643\left(a_{1}\right)$ & $\begin{array}{l}643 \\
655\end{array}$ & $584 ?[2], 617[6], 615[7]$ \\
\hline $673\left(a_{1}\right)$ & $\begin{array}{l}673 \\
675\end{array}$ & $672[2], 655[6], 655[7]$ \\
\hline
\end{tabular}

aThe assignments of the observed ground state IR vibrations is based on the symmetry of normal vibrations as specified in [6], while the vibrations from the Raman spectrum [7] and vibronic transitions from the excitation spectrum of dimer fluorescence [2] are assigned by the similarity of their frequency to the calculated frequency of normal vibrations.

The presence of additional low-frequency modes in (ACD) $)_{2}$ is expected and they must be of intermolecular origin - six additional intermolecular vibrations are expected to arise when two ACD molecules come together and form (ACD) 2 dimer in a supersonic jet. In the first approximation the frequencies of intermolecular modes depend on the binding energy of molecular complex. In the case of Van der Waals complex or hydrogen bonded homodimers their binding energies 
are much lower than the intramolecular binding energies and as a rule the intermolecular vibrations are low-frequency modes as compared to the most of fundamental intramolecular vibrations. As a rule, only the "stretching mode" along the intermolecular coordinate, connected with a change of the main distance between molecular components (e.g. between their molecular planes) of a homo- or heterodimer may have a frequency which is comparable with frequencies of intramolecular vibrations. Thus, the calculated six low-frequency vibrational modes (with vibrational frequencies of $5,12,21,39,105$, and $113 \mathrm{~cm}^{-1}$, respectively) must be identified as vibrational frequencies of intermolecular vibrations of (ACD) 2 . Such an assignment is further supported by the plots of computed forms of the normal modes of vibrations given in Fig. 2 which are clearly demonstrating that during these vibrations molecular counterparts of (ACD $)_{2}$ move as a whole without changes of molecular bonds or angles. The highest-frequency vibrational mode of $113 \mathrm{~cm}^{-1}$ (cf. Fig. 2) is an in-plane "stretch" normal mode of the dimer. There are

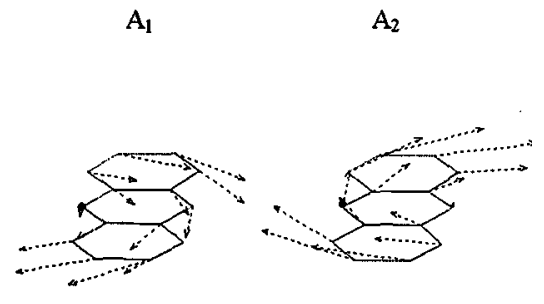

$5 \mathrm{~cm}^{-1}$

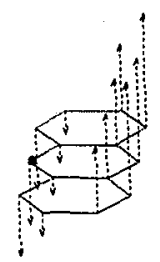

$21 \mathrm{~cm}^{-1}$

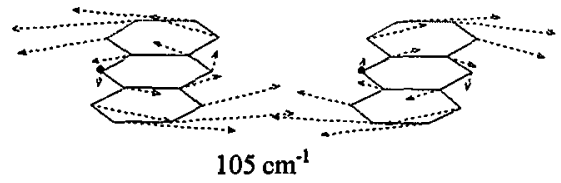

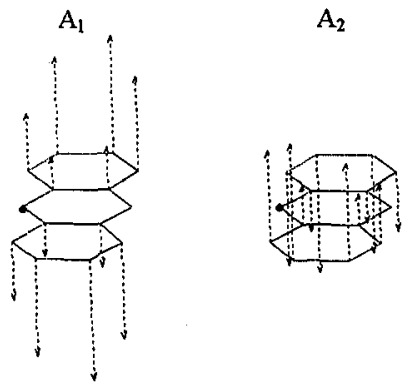

$12 \mathrm{~cm}^{-1}$

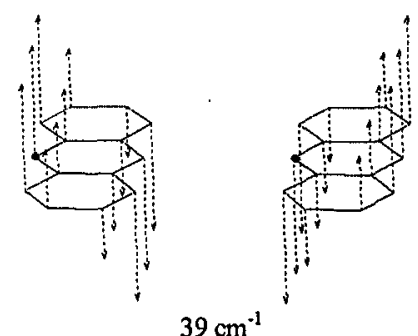

$39 \mathrm{~cm}^{-1}$

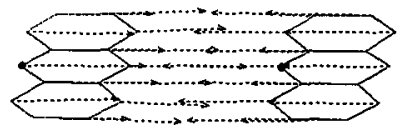

$113 \mathrm{~cm}^{-1}$

Fig. 2. Plots of the forms of normal intramolecular vibrations of acridine dimer and their harmonic frequencies (cf. Table). Acridine moieties depicted as in Fig. 1. The arrows attached to the atoms are vectors which represent their directions of motion and relative amplitudes. 
two other in-plane "bending" vibrations of 5 and $105 \mathrm{~cm}^{-1}$ vibrational frequencies. The other three vibrations $\left(12,21\right.$, and $\left.39 \mathrm{~cm}^{-1}\right)$ are clearly out-of-plane "bending" modes of the dimer.

Similar plots for the rest of normal vibrations of $(\mathrm{ACD})_{2}$, listed in Table, show clearly their intramolecular forms. This is illustrated in Figs. 3 and 4 for two arbitrary chosen normal vibrations (from low and higher vibrational frequency range). A comparison of vibrations computed for ACD molecule (also listed in Table) with intramolecular vibrations of $(\mathrm{ACD})_{2}$ shows that all normal vibrations of ACD are found in acridine dimer. However, due to the hydrogen-bond-like arrangement ( $\mathrm{C}-\mathrm{H} \cdots \mathrm{N}$ along short molecular axes direction; an $y$-axis) in the ground-state structure of the dimer, the $A_{1}$ and $A_{2}$ acridine moieties (cf. Fig. 1) are not equivalent, and any given normal vibration of ACD undergoes "splitting" into two components in the dimer. As seen in Figs. 3 and 4 in the first component the normal vibration is in phase, in both ACD molecular units, $A_{1}$ and $A_{2}$, of the dimer, while in the second component it is out of phase in both units. For all 16 normal vibrations of ACD listed in Table, a frequency difference between any given vibration of ACD molecule and a component of a higher frequency of the corresponding vibration in (ACD) $)_{2}$ is either 0 or $1 \mathrm{~cm}^{-1}$. In almost all cases under consideration the difference of frequency between the in-phase and out-of-phase. components of a given intramolecular vibration of the dimer is within the range of $1-3 \mathrm{~cm}^{-1}$ and only for three vibrations it exceeds this range (cf. 427 and 433 , 604 and 625,643 and $655 \mathrm{~cm}^{-1}$ pairs of vibrations of the dimer in Table).

Results of the present calculations are the subject to verification by the experimental data. In the case of vibrations of ACD molecule they can be compared

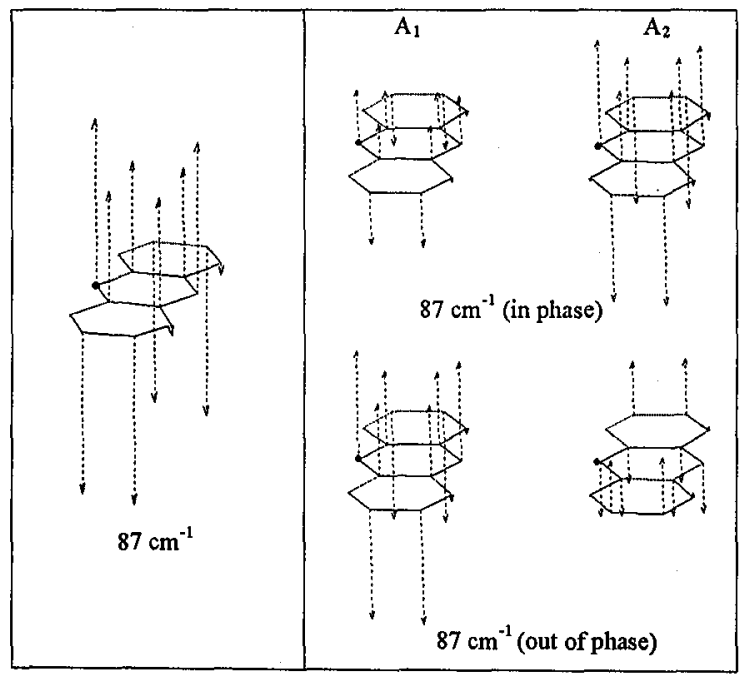

Fig. 3. Plots of the forms of normal vibration of acridine molecule (left panel) and of the components of intramolecular normal vibration of the dimer (right panel). (For explanation of other abbreviations see Fig. 2). 


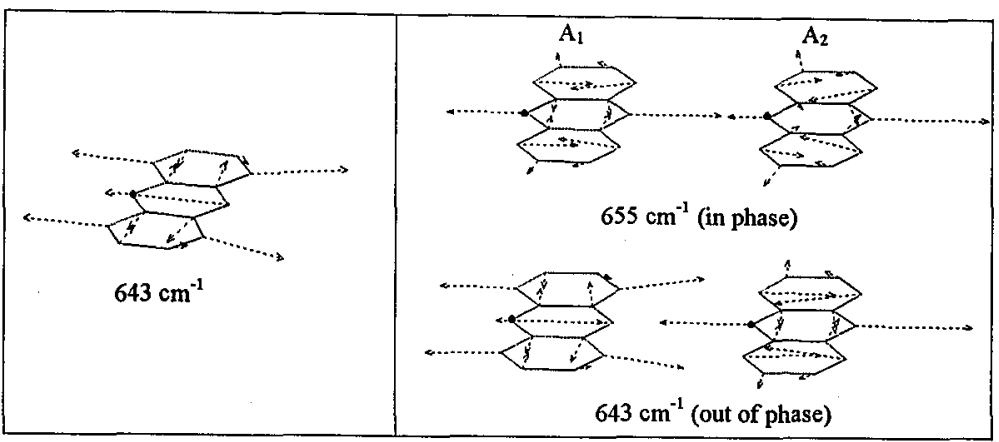

Fig. 4. Plots of the forms of normal vibration of acridine molecule (left panel) and of the components of intramolecular normal vibration of the dimer (right panel). (For explanation of other abbreviations see Fig. 2).

with the available IR and Raman spectra of ACD in liquid and solid phase [6, 7], and in Table some of these data are listed and compared with the results of the present analysis. It is seen that some of the calculated vibrational modes of ACD, e.g. $a_{1}$ 248, $a_{1} 417, a_{2} 464, b_{2} 569, b_{2} 539, b_{3} 604, a_{1} 643$, and $a_{1} 643 \mathrm{~cm}^{-1}$ (or not listed in Table $b_{1} 781 \mathrm{~cm}^{-1}$ vibration) can be identified and attributed to the earlier experimental findings $[6,7]$.

Another set of data which can be used for comparison with the above-presented calculations is contained in the LIF excitation spectrum of acridine cooled in a supersonic jet registered in our earlier experiment [2]. The excitation spectrum of LIF of acridine-seeded supersonic expansion of a helium carrier gas observed in the range of $26900-28300 \mathrm{~cm}^{-1}$ is reproduced in Fig. 5A (all experimental details of these observations were given in [2]). The transition at $27018 \mathrm{~cm}^{-1}$ has been identified as an electronic vibrationless origin of the absorption of acridine dimer, $(\mathrm{ACD})_{2}$, which is being formed in a supersonic expansion. In Fig. $5 \mathrm{~B}$ the dispersed fluorescence spectrum of $(\mathrm{ACD})_{2}$ following the excitation at $27018 \mathrm{~cm}^{-1}$ origin transition is shown. The observed emission is very weak and due to this fact it could not be used as a reliable source for the assignments although it bears all characteristics of resonant emission of the initially pumped state.

As seen in Fig. 5A the LIF excitation spectrum contains (in the range up to $\sim 200 \mathrm{~cm}^{-1}$ from the vibronic origin at $27018 \mathrm{~cm}^{-1}$ ) a number of relatively intense low-frequency vibronic transitions, with frequencies of $26,34,46,74,144$, and $152 \mathrm{~cm}^{-1}$, which could presumably be considered as the candidates for intermolecular vibrations in $(\mathrm{ACD})_{2}$. If we refer to Table, then we see that only two of the calculated intramolecular vibrations of $(A C D)_{2}$, i.e. 21 and $39 \mathrm{~cm}^{-1}$ vibrations, have frequencies relatively close to the experimental frequencies of 26 and $34 \mathrm{~cm}^{-1}$ observed in the LIF excitation spectrum of (ACD) $)_{2}$. The remaining 4 computed intermolecular vibrations (i.e. $5,12,105$, and $113 \mathrm{~cm}^{-1}$ ) do not fit to the experimental vibrations unless one would consider these latter as the overtones or combinations of the calculated normal vibrations, which may be quite fortuitous. We should remember, however, that the calculated normal modes of vibrations are corresponding to the ground state and they can be compared with 

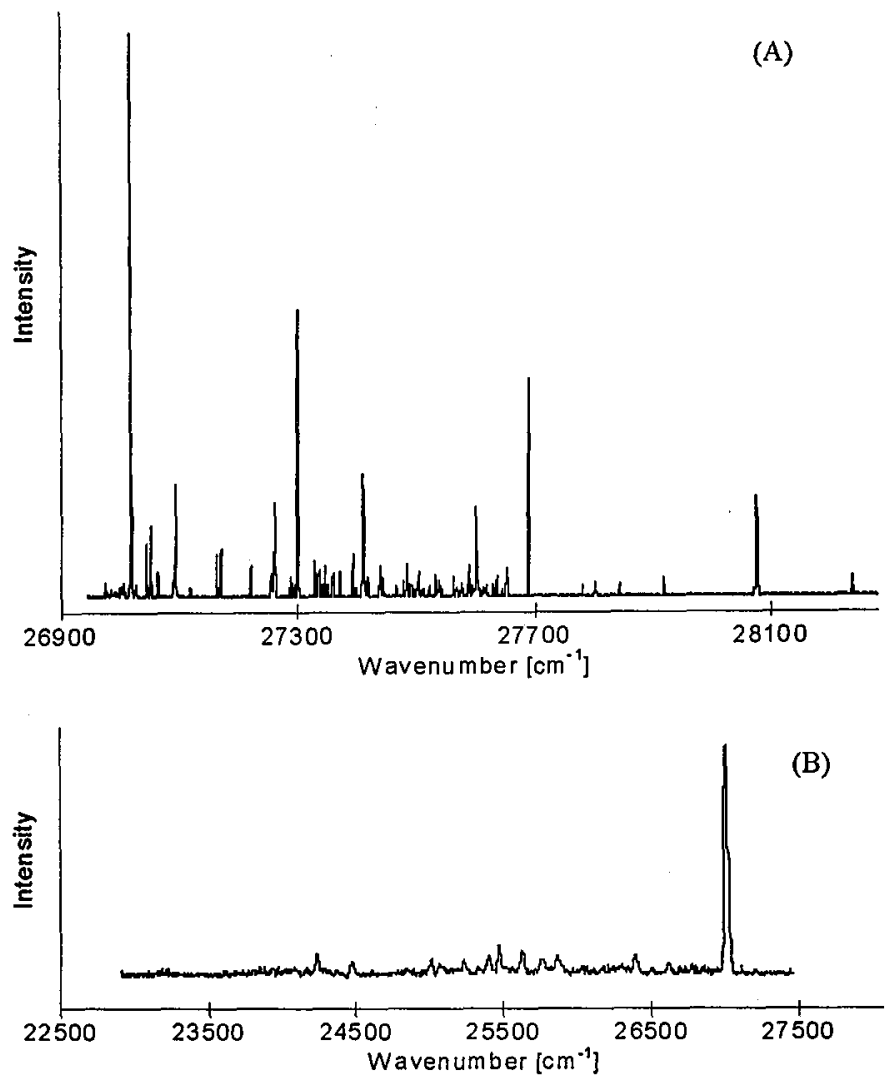

Fig. 5. (A) Excitation spectrum of laser-induced fluorescence of jet-cooled acridine dimer. The most intense transition at $27018 \mathrm{~cm}^{1}$ is the vibronic origin of the dimer. (B) Dispersed fluorescence spectrum of jet-cooled acridine dimer excited in vibronic origin (the most intense transition at $27018 \mathrm{~cm}^{-1}$ ).

the vibronic structure of the fluorescence excitation spectrum only under assumption that vibrations in the excited and in the ground state of dimer do not change remarkably in their frequency and normal mode description. There are a few known examples of large changes of vibrations (e.g. a ground-state vibration of $624 \mathrm{~cm}^{-1}$ in anthracene molecule has a frequency of $583 \mathrm{~cm}^{-1}$ in the excited state [11]) but those are rather exceptions, and by no means an apparent lack of correlation between the computed intermolecular normal vibrations and the low-frequency vibronic structure of the excitation spectrum could be considered as an evidence of the changes of the normal vibrations between the ground and excited state of $(\mathrm{ACD})_{2}$. Furthermore, since the forms of the normal vibrations in the excitation spectrum are unknown, it cannot be excluded that accidentally some of them could be just low-frequency (and of significant anharmonicity) vibrations of acridine. Without the knowledge of the optimized equilibrium structure of the dimer in the excited state, it is impossible to indicate factors which are governing and 
controlling quantitative agreement between calculations and experimental data. At that stage of the analysis the most important cause of apparent discrepancy between them is almost surely due to the well-known limitations of the PM3 method in computations of vibrational frequencies of large amplitude vibrational modes of large molecular systems [12].

On the other hand, the calculated intramolecular normal vibrations of the dimer can be relatively well related to those observed in its LIF excitation spectrum (c.f. Table), although no splitting of vibronic bands is observed in this spectrum. It must be born in mind, however, that the perturbations of intramolecular modes of $(A C D)_{2}$ dimer in the ground state (as revealed by the splittings of the vibrational modes of ACD monomer listed in Table) are in general very small which indicates a weak coupling between ACD moieties in dimer (the binding energy of equilibrium configuration of the dimer is strongly dependent on the optimization method, with the lower limit of $\sim 600 \mathrm{~cm}^{-1}$ and the upper limit of $\sim 1900 \mathrm{~cm}^{-1}$ as set by the AM1 and PM3 optimization method, respectively [2, 4]). On the other hand, in our earlier analysis of spectral characteristic of (ACD) $)_{2}$ we have found, utilizing the configuration interaction (CI) calculations, that the lowest-excited $S_{1}$ state of ACD monomer splits in (ACD) $)_{2}$ dimer into $S_{1}$ and $S_{2}$ states (this is also true for all excited singlet states of the monomer) with an energy difference of only $\sim 165 \mathrm{~cm}^{-1}$ and that $S_{0} \rightarrow S_{1}$ transition is allowed, while $S_{0} \rightarrow S_{2}$ transition is practically forbidden [4]. Hence, in view of the weak coupling between ACD moieties in the dimer and of splitting of vibronic origins (exciton splitting) which is smaller than the spacing of vibrational levels for most of the intramolecular vibrations one should not expect to observe a splitting of the vibronic bands in the fluorescence excitation spectrum of the dimer.

\section{Summary and conclusions}

The vibrational normal mode analysis of acridine dimer very clearly shows the presence of low-frequency intermolecular vibrational modes which arise due to the weak intermolecular binding of the two acridine moieties in the ground-state-stable dimer (formed upon a jet-cooling of acridine molecules). Although the forms of the intermolecular normal vibrations are physically meaningful, their quantitative agreement with the low-frequency vibronic bands observed in the laser-induced fluorescence excitation spectra of acridine dimer is only partly satisfactory which in a large part is connected with the deficiency of the semi-empirical PM3 method derived from the harmonic approximation.

In the case of intramolecular vibrational modes of the dimer the results of computations show a full correspondence (within $1 \mathrm{~cm}^{-1}$ ) with the vibrational modes computed for an acridine (monomer) molecule. However, upon the formation of dimer each vibrational normal mode of acridine undergoes (usually small) splitting into two components connected with either in-phase or out-of-phase vibrations of the two acridine moieties in dimer. Most of the computed intramolecular vibrational modes of acridine can be satisfactorily related to the vibrations which are observed either in the ground-state IR or Raman spectra of acridine in solution or in a liquid phase. Also some of the prominent vibronic bands of the excitation spectrum of laser-induced fluorescence of acridine dimer can be identified with the 
computed intramolecular vibrational modes of acridine dimer. It is clear, however, that for the quantitative analysis and assignment of the vibronic structure of the fluorescence excitation spectrum of acridine dimer observed under supersonic jet conditions it is necessary to determine the optimized structure of acridine dimer in its excited state and analyze the potential energy surfaces of the combining ground and excited state. And ultimately these must be done despite the fact that such an optimization of the excited-state structure of a large molecular system as acridine dimer is a very difficult and time-consuming computational task.

\section{Acknowledgments}

We are grateful to Dr. A. Tramer for his interest in this work and many helpful discussions. We also acknowledge partial financial support of this work by the Committee for Scientific Research under project 2P03B 08411.

\section{References}

[1] Jet Spectroscopy and Molecular Dynamics, Eds. J.N. Hollas, D. Phillips, Blackie Academic \& Professional, London 1995.

[2] J. Prochorow, I. Deperasińska, O. Morawski, Chem. Phys. Lett. 316, 24 (2000).

[3] L.A. Diverdi, M.R. Topp, J. Phys. Chem. 83, 3477 (1984).

[4] J. Prochorow, I. Deperasińska, O. Morawski, J. Mol. Struct., in press.

[5] A.G. Taylor, A.C. Jones, D. Phillips, Chem. Phys. Lett. 138, 413 (1989).

[6] M. Brigodiot, J.M. Lebas, J. Chim. Phys. Phys.-Chim. Biol. 69, 964 (1972).

[7] B. Schrader, Raman and Infrared Atlas of Organic Compounds, 2nd ed., VCH, Weinheim 1989 (entry No. I10-01).

[8] D. Narva, D.S. McClure, Chem. Phys. 56, 181 (1981).

[9] J. Prochorow, B. Kozankiewicz, Bango Bango Dongo Gemi, O. Morawski, Acta Phys. Pol. A 94, 749 (1998).

[10] J.J.P. Stewart, J. Comput. Chem. 14, 221, 230 (1989).

[11] W.R. Lambert, P.M. Felker, J.A. Syage, A.H. Zewail, J. Chem. Phys. 81, 2195 (1984).

[12] HyperChem. Computation Chemistry, Publication HC50-00-03-00, Hypercube Inc., Canada 1996. 